\title{
Heterogeneous Clusters of Phthalocyanine and Water Prepared and Probed in Superfluid Helium Nanodroplets
}

\author{
Published as part of The Journal of Physical Chemistry virtual special issue "F. Javier Aoiz Festschrift". \\ J. Fischer, ${ }^{\dagger}$ F. Schlaghaufer, ${ }^{\dagger}$ E.-M. Lottner, ${ }^{\dagger}$ A. Slenczka, ${ }^{*}{ }^{\dagger} \odot$ L. Christiansen, ${ }^{\dagger}$ H. Stapelfeldt, ${ }^{\dagger}$ M. Karra, ${ }^{\S}$ \\ B. Friedrich, ${ }^{*, \S}$ Th. Mullan, ${ }^{\perp}$ M. Schütz, ${ }^{\perp}$ T) and D. Usvyat ${ }^{*, \perp}$ \\ ${ }^{\dagger}$ Institut für Physikalische und Theoretische Chemie, Universität Regensburg, 93053 Regensburg, Germany \\ ${ }^{\ddagger}$ Department of Chemistry, Aarhus University, Langelandsgade 140, 8000 Aarhus C, Denmark \\ ${ }^{\S}$ Fritz-Haber-Institut der Max-Planck-Gesellschaft, Faradayweg 4-6, 14195 Berlin, Germany \\ ${ }^{\perp}$ Institut für Chemie, Humboldt-Universität zu Berlin, Unter den Linden 6, 10099 Berlin, Germany
}

Supporting Information

\begin{abstract}
Superfluid helium nanodroplets comprised of thousands to millions of helium atoms can serve as a reactor for the synthesis of heterogeneous molecular clusters at cryogenic conditions. The cluster synthesis occurs via consecutive pick-up of the cluster building blocks by the helium droplet and their subsequent coalescence within the droplet. The effective collision cross section of the building blocks is determined by the helium droplet size and thus exceeds by orders of magnitude that of a reactive collision in the gas phase. Moreover, the cryogenic helium environment (at $0.38 \mathrm{~K}$ ) as a host promotes the formation of metastable cluster configurations. The question arises as to the extent of the actual involvement of the helium environment in the cluster formation. The present study deals with clusters of single phthalocyanine (Pc) molecules with single water molecules. A large fluorophore such as Pc offers several sites where the water molecule can attach. The resulting isomeric variants of the Pc- $\mathrm{H}_{2} \mathrm{O}$ complex can be selectively identified by electronic spectroscopy. We compare the experimental electronic spectra of the Pc$\mathrm{H}_{2} \mathrm{O}$ complex generated in superfluid helium nanodroplets with the results of quantum-chemical calculations on the same cluster but under gas-phase conditions. The number of isomeric variants observed in the helium droplet experiment comes out the same as that obtained from our gas-phase calculations.

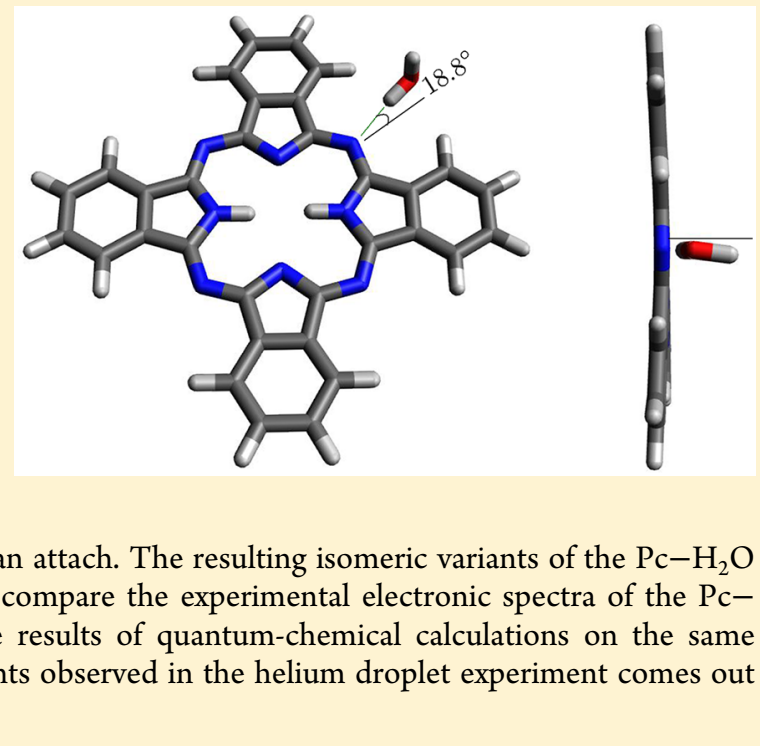

\section{INTRODUCTION}

Exploratory spectroscopic investigations have established that van der Waals clusters in helium nanodroplets form metastable configurations that are absent in the gas phase. ${ }^{1-5}$ It is not only the superfluid, high-thermal conductivity environment that gives rise to the helium-induced configurations, but also the involvement of $\mathrm{He}$ atoms as building blocks of the clusters. This is attested by the moments of inertia of dopants such as glyoxal, OCS, or sulfur hexafluoride and numerous other dopant species that exhibit a characteristic increase due to a corotating, rigidly bound helium solvation layer. ${ }^{6-12}$ Apart from the effect on the moments of inertia as revealed by rotationally resolved infrared spectra, the influence of the helium host environment on the clusters transpires in electronic spectra as helium-induced fine structure. ${ }^{13-15}$ This manifests itself in the line shape at the electronic origin known as the zero phonon line (ZPL) and in a combined excitation of the dopant molecule and the helium environment known as the phonon wing (PW). Both ZPL and PW exhibit a dopant specific spectral shape.

Helium droplets have proved to be an ideal host for designing heterogeneous clusters of well-defined stoichiometry at cryogenic temperatures. ${ }^{16}$ This is accomplished by loading the droplets with the building blocks of the desired clusters in a pick-up process: a beam of helium droplets generated by an adiabatic expansion of precooled helium $(5-30 \mathrm{~K})$ under high pressure $(10-100 \mathrm{bar})$ through a small nozzle $(5-60 \mu \mathrm{m})$ into a vacuum chamber picks up individual dopant particles on its passage through a cell filled with a gaseous dopant sample. By passing the droplet beam through a sequence of such pick-up cells, different dopant species can be loaded into the droplet where they invariably coalesce. Thus, cluster formation is significantly enhanced: first, by the effective collision cross

Received: July 31, 2019

Revised: October 23, 2019

Published: October 31, 2019 
section, which corresponds, roughly, to the geometric cross section of the helium nanodroplet; and, second, by coalescence of the building blocks, which occurs with nearly unit probability upon pick-up. The heat intake connected with the pick-up of the dopant particles and with their coalescence inside the droplet is let out by evaporation of the droplets' helium atoms into vacuum. The temperature of the helium droplets settles at $0.38(1) \mathrm{K}$, independent of the dopant species and the droplet size. ${ }^{16}$ The pick-up process obeys Poisson statistics, which allows to assign each observed cluster signal to a given stoichiometry. ${ }^{17-19}$ In the case of heterogeneous clusters, the cluster configuration can be manipulated to some extent by choosing the pick-up sequence of the clusters' building blocks. Hence a helium droplet can be regarded as a cryogenic catalyst for the design of stoichiometrically well-defined clusters.

Investigations of molecular dynamics inside helium droplets revealed that the conditions therein are less gentle than expected for helium's superfluid phase. A benchmark experiment on the photolysis of iodomethane and perfluorated iodomethane inside helium droplets demonstrated that the dissociation dynamics known from the corresponding gasphase process is obscured by inelastic collisions of the photofragments with the helium environment. ${ }^{20-22}$ Another example is 3-hydroxyflavone, which undergoes unhindered excited state intramolecular proton transfer (ESIPT) in helium droplets, but its vibrational fine structure in the electronic spectrum is completely masked. ${ }^{23}$ Further examples along with some generalizations can be found in ref 24 . In this regard, the line shape at the electronic origin of molecular species doped into helium droplets is an important source of insight. Since superfluid helium has a vanishing viscosity, any dopant is expected to rotate freely, not unlike in the gas phase. Consequently, the spectral shape of any ZPL is expected to be dominated by the rotational fine structure of the dopant, with possible modifications by the surrounding corotating helium solvation layer. Up to now, only glyoxal ${ }^{6,7}$ has been found to exhibit a rotational fine structure at the electronic origin in helium droplets smaller than 5000 atoms. For larger droplets, the rotational fine structure is obscured by pronounced line broadening. ${ }^{25}$ Recent investigations of the ZPL at the electronic origin of phthalocyanine and porphine led to the conclusion that inhomogeneous line broadening due to the droplet size distribution is responsible for the asymmetric line shape found. ${ }^{26,27}$ Simulations of the line shape by means of the excluded volume model developed for molecules embedded in a polarizable environment suffice to reproduce the experimental line shape without including particular spectral features of the rotational band system. ${ }^{28,29}$ Apparently, molecular rotation is readily resolved in helium droplets when coupled to vibrational excitation. ${ }^{30,31}$ However, upon coupling to electronic excitation, helium-induced perturbations mask spectral contributions expected for a free rotation of the dopant species.

As a first step toward elucidating the contribution of free rotation to the $\mathrm{ZPL}$ at the electronic origin, we investigated $\mathrm{Pc}-\left(\mathrm{H}_{2} \mathrm{O}\right)_{n}$ clusters generated in helium droplets. The attachment of water molecules breaks the high symmetry of Pc and thereby transforms the rotor type from an almost symmetric top to an asymmetric top. This change might be observable in the spectral line shape. Our experimental approach has been supplemented by high-level quantum chemical calculations on all possible stable and metastable configurations of $\mathrm{Pc}-\mathrm{H}_{2} \mathrm{O}$ clusters, however only under gas phase conditions. Both the helium droplet experiment and the gas-phase quantum chemical calculations have identified three stable isomeric variants of $\mathrm{Pc}-\mathrm{H}_{2} \mathrm{O}$.

\section{EXPERIMENTAL SECTION}

The helium droplet apparatus consists of two differentially pumped vacuum chambers that are connected by a conically shaped wall which carries a trombone-shaped skimmer with an opening diameter of $2 \mathrm{~mm}$. The first chamber, pumped by an oil diffusion pump $(6000 \mathrm{~L} / \mathrm{s}$, Leyboldt) backed by a Roots blower (RUWAC 250, Leyboldt) and a vane pump (TRIVAC 65 , Leyboldt), houses the source of the helium droplet beam. The continuous-flow nozzle was designed following reference. $^{32}$ The nozzle with a $5 \mu \mathrm{m}$ diameter is attached to a closed-cycle cryostat (Sumitomo). The system is operated at a temperature of $11 \mathrm{~K}$ and a stagnation pressure of 20 bar. Thereby, a helium droplet beam could be generated consisting of a log-normal droplet size distribution of an average size of $20000 \mathrm{He}$ atoms. ${ }^{16}$

The second chamber houses a two-compartment pick-up unit for consecutive doping of the helium droplets as well as a fluorescence detection setup. The pick-up unit is fashioned with openings of about $3 \mathrm{~mm}$ diameter for the passage of the helium droplet beam. The first compartment is a stainless steal cylinder of $3 \mathrm{~cm}$ diameter wrapped by a resistive heater. This unit allows for sublimation of solid samples-Pc in the present case-whose number density is controlled by the heating power. The heater is thermally shielded by a brass cylinder attached to a liquid-nitrogen Dewar. The second compartment, $4 \mathrm{~cm}$ downstream, consists of a stainless steal tubing of about 1 $\mathrm{cm}$ diameter, which is connected to a supply of gaseous samples from outside the machine. The number density is controlled by a metering valve. The entire pipe system including the metering valve is heated in order to preclude water condensation. A cylindrical glass reservoir holding doubly distilled water is cooled by an ice-acetone bath in order to reduce the vapor pressure to values conducive to single-molecule doping conditions.

The fluorescence detection setup is mounted about $8 \mathrm{~cm}$ downstream from the pick-up unit. A laser beam entering and exiting through Brewster windows intersects the droplet beam at right angles. The optical axis of the collection optics is mounted at right angles to both the droplet and laser beams. A single quartz lens with a focal length of $6 \mathrm{~cm}(f \#=2)$ is fixed at a distance equal to its focal length from the intersection volume of the droplet and laser beams. The collected light is imaged either onto the photocathode of a PMT (model 94302 Hamamatsu) or the entrance slit of a grating spectrograph (LOT ORIEL) equipped with a charge-coupled device (CCD) camera (Andor, $256 \times 1024$ pixel) and a 1,200 line $/ \mathrm{mm}$ grating.

At the end of the vacuum chamber along the droplet beam axis a quadrupole mass spectrometer (QMS, Transpector 300, Leyboldt, resolution of $1 / 300$ ) is mounted. It allows one to monitor the quality of the helium droplet beam as well as to check the relative number density in the second compartment of the pick-up unit.

The signal from the PMT is amplified (SRS445) and fed into a photon counter (SRS400). The photon counter and the CCD detector are remote-controlled by the same computer. At a wavenumber of $15000 \mathrm{~cm}^{-1}$, the resolution of the CCD chip is about $0.7 \mathrm{~cm}^{-1}$ per pixel. The laser system used in this 
experiment was a cw ring-dye-laser (Coherent 899 Autoscan) operated in single mode with a bandwidth of less than $1 \mathrm{MHz}$.

For the experiments reported herein, the oven temperature was optimized for single molecule doping of the corresponding Pc derivative. Phthalocyanine derivatives were purchased (Aldrich, 98\% purity) and used without further purification. Doubly distilled water was used as provided by the Regensburg Chemistry Department.

\section{RESULTS AND DISCUSSION}

The fluorescence excitation spectrum of helium nanodroplets doped sequentially with $\mathrm{Pc}$ and water recorded with a frequency step-width of about $0.167 \mathrm{~cm}^{-1}$ is shown in Figure 1. Pc was picked up first, under conditions optimized for single

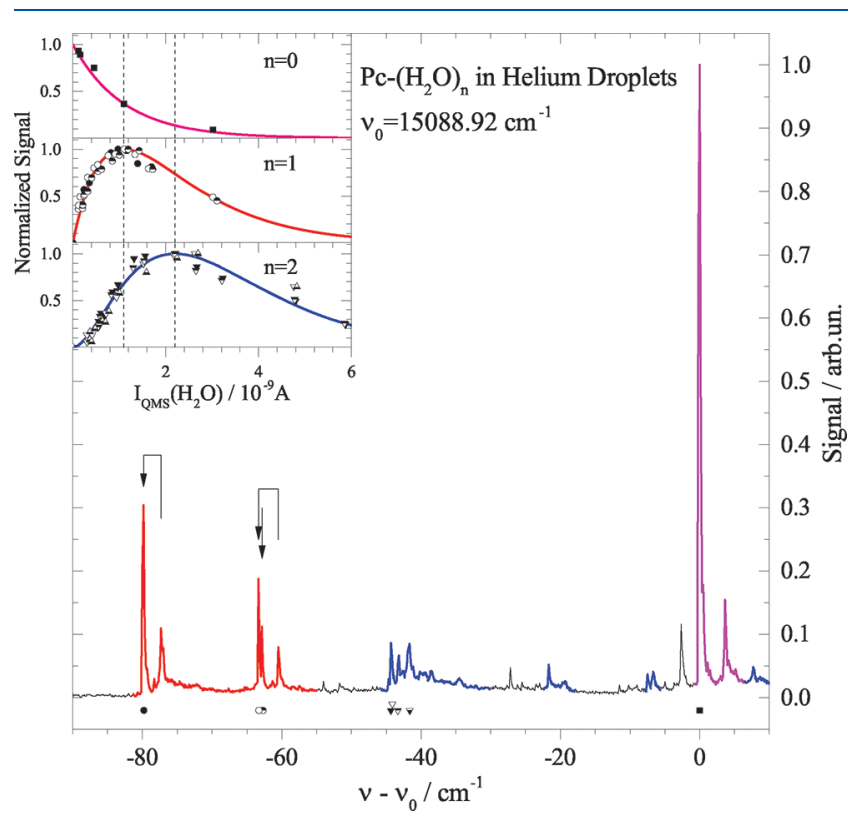

Figure 1. Overview fluorescence excitation spectrum of $\mathrm{Pc}$ in helium droplets with additional doping with water. The ZPL and PW at the electronic origin (magenta) is preceded by signals of $\mathrm{Pc}-\mathrm{H}_{2} \mathrm{O}$ (red) and $\mathrm{Pc}-\left(\mathrm{H}_{2} \mathrm{O}\right)_{2}$ (blue) clusters. Additional signals (black) can be assigned to $\mathrm{Pc}-\left(\mathrm{N}_{2}\right)_{n}$ and $\mathrm{Pc}-\left(\mathrm{O}_{2}\right)_{n}$ clusters. Black arrows mark peak positions of ZPL, in two cases linked to the accompanying PW (black vertical lines). The abscissa was shifted to $\nu_{0}=15088.92 \mathrm{~cm}^{-1}$. Insets: Poisson curves for signals of clusters without water (top, magenta, $n=$ 0 ), with one water molecule (middle, red, $n=1$ ), and with two water molecules (bottom, blue, $n=2$ ). In addition the intensity profiles of the cluster signals are added as dots that mark the relation to the corresponding peak in the cluster spectrum. The dashed vertical line marks the peak of the Poisson function for single and double pick-up of water.

particle doping. Subsequently, water was picked up, under conditions optimized for dual particle doping. According to the Poisson statistics, a fraction of $13 \%$ droplets remains free of water, $27 \%$ are doped with one or two water molecules, respectively, $18 \%$ with three, $9 \%$ with four and the residual $6 \%$ with five or more. In Figure 1 a spectral range of $100 \mathrm{~cm}^{-1}$ is shown with the abscissa shifted to the electronic origin of bare Pc in helium droplets at $15088.92 \mathrm{~cm}^{-1}$. Within $90 \mathrm{~cm}^{-1}$ to the red, the signals due to the water clusters were recorded.

In order to determine the stoichiometry of the cluster assigned to each signal, intensity profiles were recorded as functions of water particle density in the pick-up unit. Thereby, the signal intensity of the QMS at 18 amu was taken as a linear measure of the particle density. As shown in Figure 1, the corresponding profile could be unequivocally assigned to a Poisson function either without water (top inset, magneta, $n=$ 0 ), or with a single water molecule (middle inset, red, $n=1$ ), or two water molecules (bottom inset, blue, $n=2$ ). The shapes of the dots reappear below the corresponding selected peak in the fluorescence excitation spectrum of Figure 1 as do the colors for the entire set of peaks pertaining to the three cluster sizes.

In what follows, we focus on the set of the signals due to $\mathrm{Pc}-\mathrm{H}_{2} \mathrm{O}$, which are all found within a single spectral range shown in Figure 1 in red. There are a total of five resolved peaks within this range, some corresponding to electronic origins, others to PW or vibronic excitations. Excitation of a PW or a vibronic transition in helium droplets is followed by dissipation of the phonon or vibrational excess excitation energy prior to radiative decay. ${ }^{33-35}$ Consequently, emission occurs only red-shifted with respect to these excitation frequencies. However, emission coincident with the excitation frequency is indicative of no such excess excitation energy and, thus, points to an electronic origin. Accordingly, three out of the five peaks, marked by black vertical arrows, are identified as $0_{0}^{0}$ transitions representing the ZPL of three different isomeric variants of $\mathrm{Pc}-\mathrm{H}_{2} \mathrm{O}$ clusters, while the other two, marked by black vertical lines, show emission that coincides with one of the ZPLs as indicated by the horizontal links (cf. Figure 1). Because of the blue shift, these two lines represent the accompanying PW of the corresponding ZPL.

The signals of $\mathrm{Pc}-\left(\mathrm{H}_{2} \mathrm{O}\right)_{2}$ clusters plotted in blue in Figure 1 have been assigned only stoichiometrically.

A summary of the spectral properties of bare Pc and the Pc$\left(\mathrm{H}_{2} \mathrm{O}\right)_{n}$ clusters $(n=1,2)$ shown in Figure 1 is given in Table 1. This includes the frequency position and the water-induced

Table 1. Spectral Properties of Bare Pc and of the Pc$\left(\mathrm{H}_{2} \mathrm{O}\right)_{n}$ Clusters, with $n=1,2^{a}$

$\begin{array}{lccll}n & \nu, \mathrm{cm}^{-1} & \nu-\nu_{0}, \mathrm{~cm}^{-1} & \text { rel int. } & \text { assignment } \\ 0 & 15092.7 & 3.8 & 0.05 & \mathrm{PW}^{36} \\ 0 & 15089.5 & 0.6 & 0.09 & \text { ZPL } 0_{0}^{036} \\ 0 & 15088.9 & 0 & 1 & \text { ZPL } 0_{0}^{036} \\ 1 & 15028.5 & -60.4 & 0.06 & \text { PW } \\ 1 & 15026.2 & -62.7 & 0.14 & \text { ZPL } 0_{0}^{0} \\ 1 & 15025.6 & -63.3 & 0.32 & \text { ZPL 0 } \\ 1 & 15011.8 & -77.1 & 0.06 & \text { PW } \\ 1 & 15009.1 & -79.8 & 1 & \text { ZPL } 0_{0}^{0} \\ 2 & 15047.3 & -41.6 & 1 & (\mathrm{ZPL}) \\ 2 & 15046.5 & -42.4 & 0.46 & \text { (ZPL) } \\ 2 & 15045.6 & -43.3 & 0.44 & \text { (ZPL) } \\ 2 & 15044.8 & -44.1 & 0.44 & \\ 2 & 15044.6 & -44.3 & 0.86 & \text { (ZPL) }\end{array}$

${ }^{a}$ Here $\nu$ is the frequency position and $\nu-\nu_{0}$ the water-induced shift. Also shown are relative intensities within the signals corresponding to each cluster size as well as assignment to ZPL or PW (see text).

frequency shift with respect to the ZPL at the electronic origin of bare Pc as well as the assignment of the observed spectral lines to either PW or ZPL.

Apart from the water-induced red shift, it is also the line shape of the ZPL at the electronic origin of the various clusters that provides valuable information. In particular, similaritiesor a lack thereof-to the line shape of the corresponding transition of bare $\mathrm{Pc}$ in helium droplets are of interest. 
Therefore, each line identified as ZPL has been also recorded with high spectral accuracy at a frequency step width of 0.01 $\mathrm{cm}^{-1}$. The resulting fluorescence excitation spectra are shown in Figure 2 for bare $\mathrm{Pc}$ and $\mathrm{Pc}-\mathrm{H}_{2} \mathrm{O}$ and in Figure 3 for Pc-

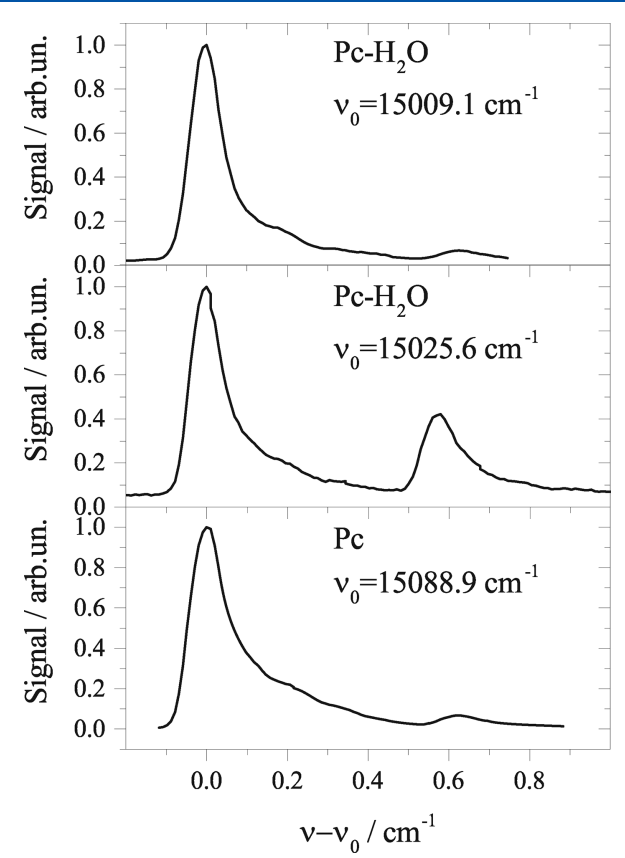

Figure 2. High precision fluorescence excitation spectrum of $\mathrm{Pc}$ and $\mathrm{Pc}-\mathrm{H}_{2} \mathrm{O}$ in helium droplets. Three spectral sections show the electronic origin of bare $\mathrm{Pc}$ in helium droplets (bottom panel), and of the three isomeric variants of the $\mathrm{Pc}-\mathrm{H}_{2} \mathrm{O}$ cluster (middle and top panel).

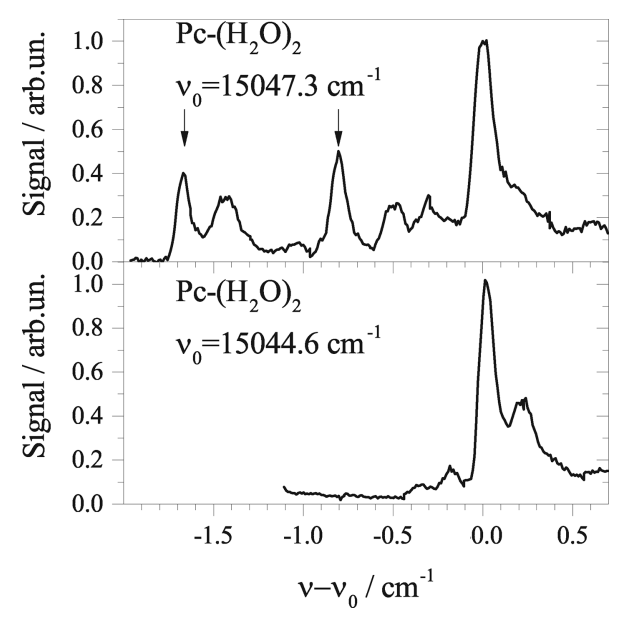

Figure 3. High precision fluorescence excitation spectrum of $\mathrm{Pc}-$ $\left(\mathrm{H}_{2} \mathrm{O}\right)_{2}$ in helium droplets.

$\left(\mathrm{H}_{2} \mathrm{O}\right)_{2}$. The line shapes of all three $\mathrm{Pc}-\mathrm{H}_{2} \mathrm{O}$ clusters (top and middle panel in Figure 2) show a similar asymmetry as recorded for bare Pc (bottom panel in Figure 2) in helium droplets. As discussed in detail in refs 26, 28, and 29, this asymmetry can be quantitatively explained by inhomogeneous line broadening due to the log-normal size distribution of the helium droplets as present in the helium droplet beam. Apparently, the attachment of a single water molecule to Pc does not significantly alter the helium-induced fine structure. This is indicative of a helium-induced effect that arises from the volume of the droplet rather than the nonsuperfluid solvation layer.

As noted above, the signals due to the $\mathrm{Pc}-\left(\mathrm{H}_{2} \mathrm{O}\right)_{2}$ clusters were not tested for $0_{0}^{0}$ transitions. Nevertheless, the three peaks in the upper panel of Figure 3 (two of which are marked by arrows and the most intense peak is at $0 \mathrm{~cm}^{-1}$ ), and the prominent peak in the bottom panel of the same figure exhibit also similar line shapes as the $0_{0}^{0}$ peak of bare Pc shown in the bottom panel of Figure 2. Note that all the other signals shown in Figure 3 have quite different spectral shapes from those of Figure 2. In this particular case, the line shape might be indicative of the ZPL at the electronic origin. However, the criterion of "similar line shape" provides only a tentative assignment.

While trying to deduce the effect of a helium environment, we see that the spectral signature shown in Figure 1 does not relate to the corresponding spectrum recorded under gas-phase conditions (cf. Figure 6 of ref 37). In the gas phase, the largest water-induced red-shifted signal, at $-71.2 \mathrm{~cm}^{-1}$, was assigned to the $0_{0}^{0}$ transition of the $\mathrm{Pc}-\mathrm{H}_{2} \mathrm{O}$ cluster-not too dissimilar from the largest water induced red-shift of $-79.8 \mathrm{~cm}^{-1}$ in helium droplets. However, the second largest red-shifted signal, lying $15.7 \mathrm{~cm}^{-1}$ to the blue of the $0_{0}^{0}$ signal was assigned to an $\mathrm{A}_{0}^{1}$ transition of the same cluster. Conversely, in helium droplets an analogous signal lying $16.5 \mathrm{~cm}^{-1}$ to the blue of the largest red-shifted signal $\left(15009.1 \mathrm{~cm}^{-1}\right)$ is unequivocally identified as the ZPL of an electronic origin, and thus identified as a configurational variant of $\mathrm{Pc}-\mathrm{H}_{2} \mathrm{O}$. None of the other low-energy modes discussed in ref 37 have been observed in helium droplets. The absence of such lowenergy/large-amplitude modes might result from a damping caused by the helium environment. Lastly, we note that the water induced red-shift $\left(-71.2 \mathrm{~cm}^{-1}\right)$ lies almost at the center of the spectral range of the three $\mathrm{Pc}-\mathrm{H}_{2} \mathrm{O}$ signals in helium droplets, indicative of the fact that the net effect due to helium can add to or take away from the excitation energy depending on the location of the water moiety.

In summary, our spectroscopic investigation of $\mathrm{Pc}-\mathrm{H}_{2} \mathrm{O}$ clusters generated and probed in helium droplets reveals the presence of three isomeric variants. Given that Pc is a large, nonpolar fluorophore whose surface abounds, by chemical intuition, in sites suitable for the attachment of an atom or a small molecule such as water, the number of stable sites as observed in the experiment-three-appears to be rather low. This is even more surprising in light of the anticipated stabilizing effect of the helium environment that favors metastable cluster configurations. Apart from other case studies, a recent investigation of anthracene-argon clusters $\left(\mathrm{AN}-\mathrm{Ar}_{n}\right)$ in helium droplets revealed numerous isomers of clusters with $n$ up to five Ar atoms, in contrast to only a single configuration in the corresponding gas-phase experiment. ${ }^{38}$ Therefore, we carried out a high-level quantum chemical calculation to elucidate the nature of the Pc-water interaction.

\section{GAS-PHASE AB INITIO CALCULATIONS ON THE $\mathrm{PC}-\mathrm{H}_{2} \mathrm{O}$ COMPLEX}

Since an $a b$ initio study of a Pc-water cluster in a helium environment would be hardly feasible, we resorted to gaining insight into the cluster's most stable configurations via a gasphase calculation. In order to screen the potential energy surface and pin down its minima, we placed a water molecule at random points on and around the phtalocyanine backbone and first optimized the structures at a density-functional theory 
Table 2. Rotational Constants and Dipole Moments of the Geometry-Optimized Ground States of the Three Isomers Aligned along the Principal Axes ${ }^{a}$

\begin{tabular}{|c|c|c|c|c|c|c|c|c|c|}
\hline & \multicolumn{9}{|c|}{ in $\times 10^{-3} \mathrm{~cm}^{-1}$, Debye, and $\mathrm{cm}^{-1}\left(\mathrm{kcal} \mathrm{mol}^{-1}\right)$} \\
\hline & A & B & $\mathrm{C}$ & $\mu_{a}$ & $\mu_{b}$ & $\mu_{c}$ & $|\mu|$ & B.E. & $\Delta$ B.E. \\
\hline Side-pocket & 2.929 & 2.644 & 1.394 & -2.05 & 0.24 & 0.73 & 2.19 & $-2068(-5.91)$ & 0 \\
\hline Center & 2.904 & 2.888 & 1.476 & 0.19 & -0.00 & -2.21 & 2.22 & $-1455(-4.16)$ & $613(1.75)$ \\
\hline Center-unhinged & 2.877 & 2.871 & 1.469 & 0.27 & -0.06 & -1.81 & 1.84 & $-1420(-4.06)$ & $648(1.85)$ \\
\hline
\end{tabular}

${ }^{a}$ Counterpoise corrected MP2/aug-cc-pVDZ absolute binding energies (see eq 1) and the relative stabilities with respect to the most stable configuration, are appended in the final two columns.

level using the BP- $86^{39}$ exchange-correlation functional along with an additional Grimme-type dispersion ${ }^{40}$ correction (D2) as implemented in the Molpro package. ${ }^{41}$ This resulted in four probable configurations which were then subjected to MP2 (aug-cc-pVDZ basis set) level of theory with the density fitting approximation (also called the RI approximation as implemented in the Turbomole suite ${ }^{42}$ ) in order to obtain their optimized geometries in the electronic ground state. Three of the MP2 optimizations were found to converge to unique structures, while the fourth one converged into one of the previous three. Their counterpoise corrected interaction energies were evaluated on the optimized structures using the expression

$$
\text { B. } \begin{aligned}
\mathrm{E} . & =E(\mathrm{AB})-E\left(\mathrm{~A}^{\mathrm{dim}} \mathrm{B}_{\text {ghost }}^{\mathrm{dim}}\right)-E\left(\mathrm{~A}_{\text {ghost }}^{\mathrm{dim}} \mathrm{B}^{\mathrm{dim}}\right) \\
& +E\left(\mathrm{~A}^{\mathrm{dim}}\right)-E\left(\mathrm{~A}^{\mathrm{opt}}\right)+E\left(\mathrm{~B}^{\mathrm{dim}}\right)-E\left(\mathrm{~B}^{\text {opt }}\right),
\end{aligned}
$$

where $E$ is the total energy (in our case MP2/aug-cc-pVDZ), A and $\mathrm{B}$ refer to the monomers ( $\mathrm{Pc}$ and water), and the superscripts "dim" and "opt" denote the monomer either in the dimer geometry or in a free optimized one, respectively. The monomers denoted as "ghost" hold only the corresponding basis functions (no nuclear charge, no electrons). The last four terms can together be interpreted as the structure relaxation energy.

The rotational constants and dipole moments of the three converging configurations are summarized in Table 2 . Contrary to simple chemical intuition gleaned from classical potentials, the side-pocket configuration, as illustrated in Figure $4 a$, appears to be energetically more favored than the symmetric-at-first-glance center configuration that has been reported in a previous paper. $^{37}$ The center-unhinged configuration is essentially a relaxed version of the center configuration with one of the two hydrogen bonds severed, however both are virtually isoenergetic. Furthermore, the

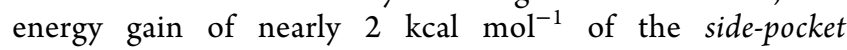
configuration with respect to the other two is quite substantial, especially at the scale of the overall interaction energy of 4-6 $\mathrm{kcal} \mathrm{mol}^{-1}$. Although the level of theory is just MP2, with a relatively small basis set, it is unlikely that a higher-level treatment can invert this picture.

The presence of the water moiety at specific locations somewhat distorts the planarity of the Pc molecule in nontrivial ways. However, the relative relaxation energies of the center and center-unhinged configurations with respect to the side-pocket are small: on the order of $0.1 \mathrm{kcal} \mathrm{mol}^{-1}$. The process of complex formation affects the geometry of the water molecule too, the details of which are summarized in Table 3. But in general, the $\mathrm{Pc}$-water potential energy surface is quite shallow and, in particular, virtually flat over the four benzorings on the periphery of the phthalocyanine molecule. This

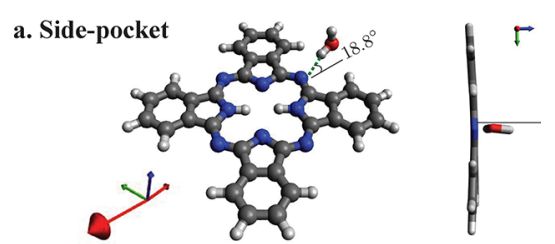

b. Center
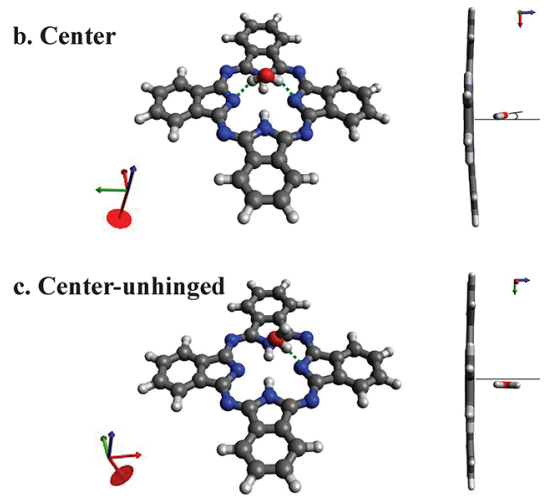

Figure 4. Three isomeric configurations of the gas-phase $\mathrm{Pc}-\mathrm{H}_{2} \mathrm{O}$ cluster arranged from top to bottom in an order of decreasing overall relative stabilities. The principal axes of the system, $a b c$, are indicated in red, green, and blue respectively, in addition to a dipole moment vector. Hydrogen bonds are indicated with green dashed lines. Table. 3 provides the values of the two relevant dihedral angles for isomers a and $c$.

readily explains the fourth configuration sliding into the known configuration, center-unhinged, and provides a sound rationale for the lack of further stable isomeric configurations-a phenomenon that may be unique to the $\mathrm{Pc}-\mathrm{H}_{2} \mathrm{O}$ system.

All three configurations are asymmetric top rotors with $C_{1}$ symmetry. On the basis of the values of the rotational constants, the asymmetry parameter, $\kappa=(2 B-A-C) /(A-$ $C)$, is equal to $0.629,0.978$, and 0.991 for side-pocket, center, and center-unhinged, respectively. The center configuration is symmetric about a vertical mirror plane passing through the two inner hydrogen atoms of the Pc moiety and containing the oxygen atom of the water moiety. An orthogonal plane contains the two $\mathrm{N}::: \mathrm{H}$ hydrogen bonds, while the oxygen atom tilts out of the plane. In contrast, the side-pocket and center-unhinged configurations possessing only one hydrogen bond each, preclude the presence of any symmetry elements. In addition to the relevant dihedral tilts of the inner and the outer $\mathrm{O}-\mathrm{H}$ bonds (see Table 3 ) of the water moiety, in both of these configurations, the $\mathrm{N}::: \mathrm{H}$ hydrogen bonds themselves show a minute dihedral tilt with respect to the $\mathrm{N}-\mathrm{N}$ planes perpendicular to the Pc moiety, i.e., the inner hydrogen atom of the water moiety lies out of the said plane. Thus, from the asymmetry in all three optimized structures, we surmise the existence of equivalent tunnelling doublets occupying the 
Table 3. Properties of the Water Moiety Attached to a Phthalocyanine Molecule in Three Different Configurations ${ }^{a}$

\begin{tabular}{|c|c|c|c|c|c|c|}
\hline & \multicolumn{6}{|c|}{ bond lengths in $\AA$; bond angles in deg } \\
\hline & $\mathrm{N}::: \mathrm{H}$ & $\mathrm{N}-\mathrm{N}::: \mathrm{H}_{i}-\mathrm{O}$ dihedral angle & $\mathrm{N}::: \mathrm{H}_{i}-\mathrm{O}-\mathrm{H}_{o}$ dihedral angle & inner $\mathrm{O}-\mathrm{H}$ & outer $\mathrm{O}-\mathrm{H}$ & $\mathrm{H}-\mathrm{O}-\mathrm{H}$ angle \\
\hline side-pocket & 1.943 & 149.8 & -158.1 & 0.981 & 0.966 & 104.4 \\
\hline center & 2.249 & - & - & 0.971 & 0.971 & 102.9 \\
\hline center-unhinged & 1.990 & -10.5 & -173.2 & 0.975 & 0.966 & 104.5 \\
\hline
\end{tabular}

${ }^{a}$ Subscripts " $i$ " and " $o$ " refer to the inward-facing and outward-facing hydrogen atoms of the water molecule, with respect to the hydrogen-bonded nitrogen atom of the Pc moiety. The dihedral angle $\mathrm{N}::: \mathrm{H}-\mathrm{O}-\mathrm{H}::: \mathrm{N}$ is $0^{\circ}$ for the center configuration, indicating that the two hydrogen bonds are co-planar.

minima of a double-well potential hypersurface for each of these three configurations.

To summarize, the theoretical approach reveals three isomeric clusters, albeit in the gas-phase. Each differs in the docking position of the water molecule on the Pc backbone. Adding to the existing literature on the single water moleculePc clusters in the gas-phase, we find the side-pocket configuration possessing only a single hydrogen bond to be surprisingly the most favored configuration-more stable than even the center configuration which possesses two hydrogen bonds. A closer look at the length of the hydrogen bonds and the distortions induced in the two moieties paints a stabilization gradient that supports the final result (see Supporting Information for bond-length and bond-angle data). An enhancement in the net dipole moment in two of the three configurations and a slight marginal decrease in the center-unhinged configuration compared to that of a single free water molecule $(1.85 \mathrm{D})$ is also attributed to the induced deformation in the geometries of the two moieties, and it was separately confirmed by single-point calculations on each deformed moiety bereft of the influence of the other.

\section{CONCLUSIONS}

The experiment on $\mathrm{Pc}-\left(\mathrm{H}_{2} \mathrm{O}\right)_{n}$ clusters generated in helium droplets reveals the presence of three configurational isomers exhibiting different electronic excitation energies for the Pc$\mathrm{H}_{2} \mathrm{O}$ cluster, and multiple partly overlapping signals for the $\mathrm{Pc}-\left(\mathrm{H}_{2} \mathrm{O}\right)_{2}$ cluster. The helium-induced red-shift of the transition frequencies was found to be $-62.7,-63.3$, and $-79.8 \mathrm{~cm}^{-1}$, respectively, for the three signals of the $\mathrm{Pc}_{\mathrm{c}}-\mathrm{H}_{2} \mathrm{O}$ cluster with respect to the bare $\mathrm{Pc}$ signal, showing an increasing intensity trend toward the red. The spectral shape of the electronic origin of each of these clusters is almost identical showing a rather steep edge on the red side and an extending tail indicative of inhomogeneous broadening to the blue. This asymmetry appears to be the same as that of the line shape observed at the electronic origin of bare Pc in helium droplets. ${ }^{28}$ Likewise, we rule out any possible resolution of an underlying rotational substructure. The attachment of polar water molecule(s) to the bare Pc molecule appears to have little effect-other than a shift in the transition energies. As we have shown in more detail for bare Pc and porphine, ${ }^{26,27}$ the asymmetry in these line shapes is helium-induced and reflects the inhomogeneity caused by the droplet size distribution. The perseverance of the line shape despite the attachment of a water molecule supports the idea of an effect that is caused by the droplet volume rather than just the helium solvation layer surrounding the dopant cluster.

Contrary to expectation based on a recent study of anthracene-Ar ${ }_{n}$ clusters, $^{38}$ the number of isomeric configurations predicted by theory (in the gas phase) concurs with the number of experimentally distinguishable isomers in the helium environment. However, a final assignment of the cluster configurations to the experimental signals could not yet be realized. One might speculate that the most intense of the Pc$\mathrm{H}_{2} \mathrm{O}$ signals, namely the one with the greatest helium-induced red shift, represents the most stable of the three configurations (side-pocket). Although this idea is also supported by the close adjacency of the other two signals and the possibility of an interconversion between the center and the center-unhinged configurations, a simple correlation between energetic stability and the most intense signal may be outweighed by significant differences in the oscillator strengths.

In our upcoming work, we will study the Stark spectra of the Pc-water complexes in helium droplets. The spectral contribution due to rotation is expected to be modified by the electric dipole interaction with the Stark field, leading to characteristic line shapes. The Stark field may also affect the relative internal rotation of the two moieties, if indeed present, resulting in an additional characteristic variation of the spectral line shapes.

Our complementary ongoing work concerns field-free and Stark spectra of the clusters in the gas phase. As exemplified by numerous gas-phase studies, the rotational degree of freedom is invaluable for elucidating the structure of species ranging from simple molecules to complicated clusters. ${ }^{4-45}$

On the theoretical side, a detailed study of the transition states (to determine tunnelling barriers) and electronic excitations will complement the present study by elucidating the changes in rotor properties upon electronic excitation, including the angles between the transition and permanent dipole moments of the $\mathrm{Pc}-\mathrm{H}_{2} \mathrm{O}$ cluster.

\section{ASSOCIATED CONTENT}

\section{S Supporting Information}

The Supporting Information is available free of charge on the ACS Publications website at DOI: 10.1021/acs.jpca.9b07302.

Tables listing bond-length and bond angle data as calculated for the side pocket, the center, and the center unhinged configurations of the $\mathrm{Pc}-\mathrm{H}_{2} \mathrm{O}$ cluster and, in addition, a top view of each cluster showing the numbering used for all the atoms (PDF)

\section{AUTHOR INFORMATION}

\section{Corresponding Authors}

*(A.S.) E-mail: alkwin.slenczka@chemie.uni-regensburg.de.

*(B.F.) E-mail: bretislav.friedrich@fhi-berlin.mpg.de.

*(D.U.) E-mail: denis.usvyat@chemie.hu-berlin.de.

ORCID $\odot$

A. Slenczka: 0000-0002-6594-9503

B. Friedrich: 0000-0002-1299-4239

M. Schütz: 0000-0002-2688-3214 


\section{Notes}

The authors declare no competing financial interest.

\section{ACKNOWLEDGMENTS}

This work would not have been possible without the endeavors of Prof. Martin Schütz (1963-2018). His contributions to the field of $a b$ initio electronic structure theory of extended molecular systems were seminal. A.S., J.F., B.F., and M.K. gratefully acknowledge the support by the DFG (Deutsche Forschungsgemeinschaft) through SPP1807. E.-M.L. gratefully acknowledges a doctoral fellowship from Cusanuswerk Bischöfliche Studienförderung. We would also like to thank Dr. Maristella Alessio for her help with the Turbomole program.

\section{REFERENCES}

(1) Nauta, K.; Miller, R. E. Nonequilibrium self-assembly of long chains of polar molecules in superfluid helium. Science 1999, 283, 1895-1897.

(2) Nauta, K.; Miller, R. E. Formation of cyclic water hexamer in liquid helium: the smallest piece of ice. Science 2000, 287, 293-295.

(3) Pörtner, N.; Vilesov, A. F.; Havenith, M. The formation of heterogeneous van der Waals complexes in helium droplets. Chem. Phys. Lett. 2001, 343, 281-288.

(4) Lindinger, A.; Toennies, J. P.; Vilesov, A. F. Laser-induced uorescence spectra of tetracene complexes with $\mathrm{Ne}, \mathrm{H}_{2} \mathrm{O}, \mathrm{D}_{2} \mathrm{O}$ inside He droplets. Chem. Phys. Lett. 2006, 429, 1-7.

(5) Kuma, S.; Nakahara, H.; Tsubouchi, M.; Takahashi, A.; Mustafa, M.; Sim, G.; Momose, T.; Vilesov, A. F. Laser induced fluorescence spectroscopy of tetracene with large $\mathrm{Ar}, \mathrm{Ne}$, and $\mathrm{H}_{2}$ clusters in superfluid He nanodroplets. J. Phys. Chem. A 2011, 115, 7392-7399.

(6) Hartmann, M.; Mielke, F.; Toennies, J. P.; Vilesov, A. F.; Benedek, G. Direct spectroscopic observation of elementary excitations in superfluid He droplets. Phys. Rev. Lett. 1996, 76, $4560-4563$

(7) Pörtner, N.; Toennies, J. P.; Vilesov, A. F. The observation of large changes in the rotational constants of glyoxal in superfluid helium droplets upon electronic excitation. J. Chem. Phys. 2002, 117, 6054-6060.

(8) Hartmann, M. Ph.D. Dissertation, MPI für Strömungsforschung, Bericht 10/1997; Göttingen: Germany, 1997.

(9) Grebenev, S.; Toennies, J. P.; Vilesov, A. F. Superfluidity within a small helium-4 cluster: the microscopic andronikashvili experiment. Science 1998, 279, 2083-2086.

(10) Hartmann, M.; Miller, R. E.; Toennies, J. P.; Vilesov, A. F. Rotationally resolved spectroscopy of $\mathrm{SF}_{6}$ in liquid helium clusters: $\mathrm{A}$ molecular probe of cluster temperature. Phys. Rev. Lett. 1995, 75, 1566.

(11) Harms, J.; Hartmann, M.; Toennies, J. P.; Vilesov, A. F.; Sartakov, B. Rotational structure of the IR spectra of single $\mathrm{SF}_{6}$ molecules in liquid ${ }^{4} \mathrm{He}$ and ${ }^{3} \mathrm{He}$ Droplets. J. Mol. Spectrosc. 1997, 185, 204-206.

(12) Harms, J.; Hartmann, M.; Sartakov, B.; Toennies, J. P.; Vilesov, A. F. High resolution infrared spectroscopy of single $\mathrm{SF}_{6}$ molecules in helium droplets. II. The effect of small amounts of ${ }^{4} \mathrm{He}$ in large ${ }^{3} \mathrm{He}$ droplets. J. Chem. Phys. 1999, 110, 5124-5136.

(13) Premke, T.; Wirths, E.-M.; Pentlehner, D.; Riechers, R.; Lehnig, R.; Vdovin, A.; Slenczka, A. Microsolvation of molecules in superfluid helium nanodroplets revealed by means of electronic spectroscopy. Front. Chem. 2014, 2, 51.

(14) Hartmann, M.; Lindinger, A.; Toennies, J. P.; Vilesov, A. F. The phonon wings in the $\left(S_{1} \leftarrow S\right)_{0}$ spectra of tetracene, pentacene, porphin and phthalocyanine in liquid helium droplets. Phys. Chem. Chem. Phys. 2002, 4, 4839-4844.

(15) Riechers, R.; Pentlehner, D.; Slenczka, A. Microsolvation in superfluid helium droplets studied by the electronic spectra of six porphyrin derivatives and one chlorine compound. J. Chem. Phys. 2013, 138, 244303.

(16) Toennies, J. P.; Vilesov, A. F. Superfluid helium droplets: a uniquely cold nanomatrix for molecules and molecular complexes. Angew. Chem., Int. Ed. 2004, 43, 2622-2648.

(17) Lewerenz, M.; Schilling, B.; Toennies, J. P. Successive capture and coagulation of atoms and molecules to small clusters in large liquid helium clusters. J. Chem. Phys. 1995, 102, 8191-8207.

(18) Hartmann, M.; Miller, R. E.; Toennies, J. P.; Vilesov, A. F. High-resolution molecular spectroscopy of van der Waals clusters in liquid helium droplets. Science 1996, 272, 1631-1634.

(19) Hartmann, M.; Lindinger, A.; Toennies, J. P.; Vilesov, A. F. Laser-induced fluorescence spectroscopy of van der Waals complexes of tetracene- $\operatorname{Ar}_{N}(N \leq 5)$ and pentacene-Ar within ultracold liquid $\mathrm{He}$ droplets. Chem. Phys. 1998, 239, 139-149.

(20) Braun, A.; Drabbels, M. Photodissociation of alkyl iodides in helium nanodroplets. I. Kinetic energy transfer. J. Chem. Phys. 2007, $127,114303$.

(21) Braun, A.; Drabbels, M. Photodissociation of alkyl iodides in helium nanodroplets. II. Solvation dynamics. J. Chem. Phys. 2007, 127, 114304 .

(22) Braun, A.; Drabbels, M. Photodissociation of alkyl iodides in helium nanodroplets. III. Recombination. J. Chem. Phys. 2007, 127, 114305.

(23) Lehnig, R.; Pentlehner, D.; Vdovin, A.; Dick, B.; Slenczka, A. Photochemistry of 3-hydroxyflavone inside superfluid helium nanodroplets. J. Chem. Phys. 2009, 131, 194307.

(24) Pentlehner, D.; Riechers, R.; Vdovin, A.; Pötzl, G. M.; Slenczka, A. Electronic spectroscopy of molecules in superfluid helium nanodroplets: an excellent sensor for intramolecular charge redistribution. J. Phys. Chem. A 2011, 115, 7034-7043.

(25) Pörtner, N. Ph.D. Dissertation, MPI für Strömungsforschung, Bericht 1/2001, Göttingen: Germany, 2001.

(26) Fuchs, S.; Fischer, J.; Slenczka, A.; Karra, M.; Friedrich, B. Microsolvation of phthalocyanine molecules in superfluid helium nanodroplets as revealed by the optical line shape at electronic origin. J. Chem. Phys. 2018, 148, 144301.

(27) Fischer, J.; Fuchs, S.; Slenczka, A.; Karra, M.; Friedrich, B. Microsolvation of porphine molecules in superfluid helium nanodroplets as revealed by optical line shape at the electronic origin. J. Chem. Phys. 2018, 149, 244306.

(28) Slenczka, A.; Dick, B.; Hartmann, M.; Peter Toennies, J. Inhomogeneous broadening of the zero phonon line of phthalocyanine in superfluid helium droplets. J. Chem. Phys. 2001, 115, 1019910205 .

(29) Dick, B.; Slenczka, A. Inhomogeneous line shape theory of electronic transitions for molecules embedded in superfluid helium droplets. J. Chem. Phys. 2001, 115, 10206-10213.

(30) Choi, M. Y.; Douberly, G. E.; Falconer, T. M.; Lewis, W. K.; Lindsay, C. M.; Merritt, J. M.; Stiles, P. L.; Miller, R. E. Infrared spectroscopy of helium nanodroplets: novel methods for physics and chemistry. Int. Rev. Phys. Chem. 2006, 25, 15-75.

(31) Verma, D.; Tanyag, R. M. P.; O’Connell, S. M. O.; Vilesov, A. F. Infrared spectroscopy in superfluid helium droplets. Advances in Physics: X 2019, 4, 1553569.

(32) Toennies, J. P.; Vilesov, A. F. Spectroscopy of atoms and molecules in liquid helium. Annu. Rev. Phys. Chem. 1998, 49, 1-41.

(33) Lehnig, R.; Slenczka, A. Emission spectra of free base phthalocyanine in superfluid helium droplets. J. Chem. Phys. 2003, $118,8256-8260$.

(34) Lehnig, R.; Slenczka, A. Microsolvation of phthalocyanines in superfluid helium droplets. ChemPhysChem 2004, 5, 1014-1019.

(35) Lehnig, R.; Slenczka, A. Quantum solvation of phthalocyanine in superfluid helium droplets. J. Chem. Phys. 2004, 120, 5064-5066.

(36) Lehnig, R. Anregungs und Emissionsspektroskopie von organischen Molekülen in ${ }^{4} \mathrm{He}$-Tröpfchen. Ph. D. Dissertation, Universität Regensburg: Germany, 2004. 
(37) Menapace, J. A.; Bernstein, E. R. Spectroscopic studies of phthalocyanines and their clusters with small molecules. J. Chem. Phys. $1987,87,6877-6889$.

(38) Lottner, E.-M.; Slenczka, A. Anthracene-Argon Clusters Generated in Superfluid Helium Nanodroplets: New Aspects on Cluster Formation and Microsolvation. J. Phys. Chem. A 2019, DOI: $10.1021 /$ acs.jpca.9b04138.

(39) Becke, A. D. Density-functional exchange-energy approximation with correct asymptotic behavior. Phys. Rev. A: At., Mol., Opt. Phys. 1988, 38, 3098-3100.

(40) Grimme, S. Semiempirical GGAtype density functional constructed with a long range dispersion correction. J. Comput. Chem. 2006, 27 (15), 1787-1789.

(41) Werner, H.-J.; Knowles, P. J.; Lindh, R.; Manby, F. R.; Schütz, M., et al. MOLPRO, Version 2015.10, a package of ab initio programs, (see http://www.molpro.net), 2006.

(42) TURBOMOLE, V7.0 2015, a development of University of Karlsruhe and Forschungszentrum Karlsruhe GmbH, 1989-2007, TURBOMOLE GmbH, since 2007; available from http://www. turbomole.com.

(43) Domingos, S. R.; Martin, K.; Avarvari, N.; Schnell, M. Water docking bias in [4]Helicene. Angew. Chem., Int. Ed. 2019, 58, 1125711261 .

(44) Lemmens, A. K.; Gruet, S.; Steber, A. L.; Antony, J.; Grimme, S.; Schnell, M.; Rijs, A. M. Far-IR and UV spectral signatures of controlled complexation and microhydration of the polycyclic aromatic hydrocarbon acenaphthene. Phys. Chem. Chem. Phys. 2019, 21, 3414-3422.

(45) Steber, A. L.; Pérez, C.; Temelso, B.; Shields, G. C.; Rijs, A. M.; Pate, B. H.; Kisiel, Z.; Schnell, M. Capturing the Elusive water trimer from the stepwise growth of water on the surface of the polycyclic aromatic hydrocarbon acenaphthene. J. Phys. Chem. Lett. 2017, 8, 5744-5750. 